\title{
1 Translational read-through inducing drugs for the treatment of inherited 2 retinal dystrophies
}

\section{Abstract:}

5 Introduction:

6 Inherited retinal disorders (IRDs) are the most common cause of certifiable blindness in 7 working age adults in the UK. There are currently no treatments for the majority of patients, 8 resulting in considerable morbidity with lifelong socioeconomic implications. $12 \%$ of all 9 genetic disease variants are nonsense mutations, which encode a premature termination 10 codon (PTC). The resultant transcript is either degraded through nonsense mediated decay

11 (NMD) or translated to produce a truncated protein. Nonsense suppression therapy aims to 12 bypass and allow peptide synthesis beyond the PTC, creating a full-length protein and 13 possible phenotypic rescue. The responsible agents, known as translational readthrough 14 inducing drugs (TRIDs), have been in continuous development to maximise readthrough 15 efficiency and minimise toxicity. These include aminoglycosides, aminoglycoside derivatives and non-aminoglycoside small molecule drugs and have been successfully applied to a

17 number of disease models in recent preclinical studies.

19 Areas Covered:

20 This review provides an update in the advancements of nonsense suppression therapy in the 21 treatment of IRDs, including an overview of this process and NMD, advancements in the development of TRIDs and barriers to clinical trials including drug developments, disease modelling and patient selection.

\section{Expert Opinion:}

26 Clinical trials are forthcoming for patients with IRDs to determine TRID suitability as a 27 viable therapy option. 
1 Keywords:

2

3

Aminoglycosides; genetic eye disease; inherited retinal disorders; nonsense mediated decay; nonsense mutations; nonsense suppression; PTC124; readthrough; small molecules; translation

Highlights:

- Mechanisms of nonsense mutations, nonsense mediated decay (NMD) and nonsense suppression therapy.

- $\quad$ Updates in development of translational readthrough inducing drugs (TRIDs) and NMD inhibitors.

- $\quad$ Recent applications to models of inherited retinal dystrophies.

- Improvements in drug delivery and clinical trial considerations.

\section{Introduction}

Inherited retinal disorders (IRDs) are the most common cause of certifiable blindness in working age adults in the UK [1]. There is significant clinical and genetic heterogeneity with over 250 causative genes according to RetNet (https://sph.uth.edu/retnet/sum-dis.htm).

Affected individuals have a profound loss in quality of life and there are no treatments for the majority, which represents a significant economic burden for health care systems and society $[2,3]$

The retina is at the vanguard of genetic therapeutic development due to its accessibility, immune-privilege site and high-resolution non-invasive imaging modalities. Over the past decade there have been exciting therapeutic advancements in retinal prosthesis, stem cells and gene therapy to treat these disorders. The first retinal gene therapy, Voretigene neparvovec (LUXTURNA ${ }^{\mathrm{TM}}$ ), was approved by the US Food and Drug Administration 
1 (FDA) in 2017, using the adeno-associated virus 2 (AAV2) for the treatment of biallelic

2 mutations in the RPE65 gene known to cause Leber congenital amaurosis type 2. Phase III

3 clinical trial (NCT00999609) results showed significant improvements in functional vision,

4 light sensitivity and visual function in participants for up to 3 years [4]. Despite several other

5 clinical trials underway, there are still concerns over efficacy, repeated administration,

6 immune response, and harmful off-target effects of viral approaches [5,6]. In addition,

7 conventional viral vectors such as AAVs have an upper packaging capacity of $5 \mathrm{~kb}$ which

8 excludes incorporation of larger genes such as $U S H 2 A$ and $E Y S$, which are commonly

9 mutated in Retinitis Pigmentosa patients [7]. Alternative methods of genetic treatments

10 include antisense oligonucleotides, which prevent pre-mRNA splicing by binding to and

11 inhibiting target mRNA sequences [8]. Positive preclinical results have led to 2 trials for IRD

12 patients with USH2A (NCT03780257) and CEP290 (NCT03140969) mutations [9-11], but

13 there is uncertainty about off-target effects and, for example, the residual function of Usherin

14 lacking exon $13[8,12]$.

16 Advances in whole exome and genome sequencing have increased the diagnostic rate of 17 disease-causing variants to over $70 \%$ for IRDs. It is estimated that approximately $12 \%$ of all 18 genetic diseases are caused by nonsense mutations [13]. In some genetic eye diseases such as

19 choroideremia and isolated aniridia, nonsense mutations account for $30 \%$ and $40 \%$ of all

20 cases respectively $[14,15]$. Therapies that target nonsense mutations to bypass the PTC have

21 therefore been widely explored; their modes of action and applications in eye diseases, and 22 particularly in IRDs, will be the focus of this review.

\section{Nonsense mutations and mRNA fate}

24 Nonsense mutations are single in-frame nucleotide changes that result in the formation of a stop codon - UAG, UAA or UGA - termed a premature termination codon (PTC). PTCs can 
1 also be introduced by indel frameshift mutations and splice-site variants causing defective

2 intron removal from pre-mRNA $[13,16]$ or via aberrant alternative splicing of mRNA

$3[17,18]$, however these are usually out-of-frame and not amenable to nonsense suppression.

4 During normal translation, the aminoacyl-tRNA complex binds the ribosomal A-site, the

5 amino acid is added to the peptide chain and translation continues (Fig.1 A). If a PTC-

6 containing mRNA transcript enters the ribosomal A-site, translation is prematurely

7 terminated [19]. The mRNA then meets one of two fates. Either, it is translated into a

8 truncated protein, which is often non-functional or can harm the cell in a number of ways

9 including misfolding, aggregation, or binding to cellular machinery and disrupting its

10 structure or function, or rarely causing pathological gain of function (Fig.1 B) [20,21]. Or,

11 PTC-containing mRNA transcripts are degraded by a natural cellular surveillance mechanism

12 called nonsense-mediated decay (NMD) [22] (Fig.2).

\section{Nonsense mediated decay}

14 NMD is an evolutionary conserved process that protects the cell from potentially harmful truncated proteins generated by PTCs. The NMD machinery must therefore correctly identify and degrade PTC-containing mRNA transcripts without affecting normal termination codons.

17 To achieve this, three NMD models have been proposed. The "faux UTR" model describes a reduction in termination kinetics at the PTC [23]. This occurs due to a failure of association

19 between the PTC and poly-A binding protein (PABP), which usually facilitates fast ribosomal release from the peptide chain (Fig.2 A). The exon junction complex (EJC)dependent model suggests that upstream termination prevents ribosomal displacement of EJCs, which are protein complexes situated 20-24 bp upstream of each exon-exon junction

23 (Fig.2 A). The combined model of NMD suggests the nondisplaced EJC and reduced 24 termination kinetics recruit the SURF complex (SMG1/UPF1/eRF1/eRF3), consisting of the helicase and translocase UPF1, eukaryotic release factors (eRFs) 1 and 3, and the protein 
1 kinase SMG1 (Fig.2 B). Within the SURF complex, SMG1 forms a complex with SMG8 and

29 and together with UPF2, a component of the EJC, activate UPF1 by SMG1-dependent

3 phosphorylation (Fig.2 B) [24]. Activated UPF1 is the key NMD regulator, which clears the

4 mRNA of proteins such as eRFs, as well as associating with phospho-binding proteins such

5 as SMG5, 6 and 7 to recruit deadenylation, decapping and endonucleolytic enzymes that

6 decay the mRNA [25,26] (Fig.2 B). Its importance and specificity in NMD is proven by

7 inactivating mutations or deletion of $U P F 1$, which increase the accumulation of only PTC-

8 containing transcripts $[27,28]$.

10 NMD is influenced by variations in genomic context: AUGs closer to the PTC inhibit NMD 11 compared to those at a distance $[29,30]$ and transcripts with longer open reading frames

12 (ORF) are more NMD sensitive [31]. NMD efficiency also varies across different tissues in

13 the same organism [32]. This was previously thought to be the result of differing expression

14 of NMD proteins in these tissues, however no correlation was found between protein

15 expression and NMD efficiency [32]. Finally, NMD efficiency varies across patients

16 harbouring the same mutation [33,34]. For example, Sarkar et al. found CHM transcript

17 levels ranged from $13 \%$ to $52.6 \%$ in choroideremia patients with the mutation c. $715 \mathrm{C}>\mathrm{T}$;

18 p.R239* [35]. These differences were likely due to individual factors as opposed to tissue-

19 specific factors as there was little difference in NMD or mRNA levels between skin

20 fibroblasts and induced pluripotent stem cell-derived retinal pigmented epithelium (iPSC-

21 RPE) of the same patient. Inter-individual variation did not correlate with differing expression of genes encoding the NMD machinery such as UPF1 [35]. A better understanding of the mutation, tissue and individual determinants of NMD efficiency is

24 therefore desired as it is a critical determinant for translational readthrough; if NMD activity

25 is high and transcript levels are low, readthrough is less effective. NMD efficiency could 
1 therefore act as a prognostic indicator for translational readthrough therapy once reliable

2 markers of efficiency can be discovered.

3

4

5 PTC-containing transcripts are typically degraded by NMD, reducing their availability.

6 Therefore, inhibition of NMD has been proposed as a way to increase mRNA stability and as

7 a consequence, increase the amount of substrate for TRIDs to target. Caffeine inhibits NMD

8 through the inhibition of SMG1 kinase [35,36] (Fig.2 C). It alone has been shown to rescue the

9 phenotype in fibroblasts carrying a PTC seen in the muscular dystrophy Ullrich's disease, by

10 increasing mRNA and protein levels of the defective collagen VI $\alpha 2$ [37]. NMDI1 is a

24 The inclusion of NMD inhibitors to clinical trials is eagerly awaited. Particular attention must tetracyclic compound that traps UPF1 in a hyperphosphorylated state, preventing downstream interactions with SMG5 [38] (Fig.2 D). It is specific for NMD, does not affect translation efficiency and is non cytotoxic [38]. In a mouse model of Mucopolysacchardisosis I-Hurler syndrome (MPS I-H) that carries the knock-in Idua p.W392* mutation, NMDI application with gentamicin resulted in greater readthrough and functional enzymatic activity than gentamicin alone [39]. However, NMDI1 synthesis is inefficient and technically difficult. In contrast, VG1, a structural analogue of NMDI1 with similar inhibition capability, is more easily produced and shows an improved yield [40]. Its mechanism of action is currently unknown but its application to IRDs is awaited. Amlexanox is a compound with both readthrough and NMD inhibition properties. It has been reported as a combined therapy option for PTCs causing cystic fibrosis $(\mathrm{CF})$ and recessive dystrophic epidermolysis bullosa, showing increased levels of full length proteins compared to G418 and PTC124 alone [41,42]. be paid to monitoring side effects, as there is little current literature $[35,43]$. In the MPS I-H 
1 mouse model, systemic NMDI1 application carried no systemic side effects, but the study

2 duration was only 3 days [39]. Caffeine has well known effects on bodily systems and

3 pathways. However, the doses required to achieve NMD inhibition may prohibit systemic

4 therapy and local administration of higher doses may be better tolerated.

\section{Nonsense suppression therapy}

7 During translation, the ribosomal A-site can interact with near-cognate codons [44]. At all

8 termination codons there is competition between the termination complex and near-cognate

9 aminoacyl-tRNAs. If a near-cognate tRNA outcompetes the termination complex, tRNA

10 complex translocates to the P-site and translation continues [45,46] (Fig.1 C). This occurs

11 endogenously but at a very low frequency (between 0.01 to $0.1 \%$ ), due to the three

12 dimensional configuration of the ribosome and key signalling proteins such as PABP $[47,48]$.

13 Endogenous readthrough of a PTC is more common (increasing to $<1 \%$ ) as the ribosomal

14 pausing facilitates more aminoacyl-tRNA sampling [49]. However, this is not in sufficient

15 levels to restore protein function. Nonsense suppression therapy uses translational

16 readthrough-inducing drugs (TRIDs) to efficiently identify and readthrough PTCs alone,

17 resulting in the production of full-length protein.

19 There are several genetic factors influencing the efficacy of nonsense suppression therapy.

20 These include the identity of the PTC, where TRIDs appear to have an affinity to

21 UGA $>$ UAG $>$ UAA. The identity of the nucleotides both downstream and upstream to the

22 codon is also important; the preference downstream is $C>U>A \geq G[21,50]$ and the presence of

23 adenine upstream at positions -1 or -2 to a PTC encourages its readthrough by modulating

24 mRNA structure P-site, which adjusts the competition between release factors and

25 aminoacyl-tRNAs [51,52]. However, readthrough efficiency cannot be predicted by the 
1 genetic composition of the PTC alone. This is shown by the failed readthrough of the $r d 12$

2 mouse carrying a theoretically readthrough-sensitive mutation in Rpe65 (discussed below)

3 [53]. Other factors include the availability of eRFs [54] and factors affecting translation

4 control such as the prolyl hydroxylation of the 40S subunit ribosomal protein (RPS23)

$5 \quad[55,56]$. The future clinical significance of this is unknown and further work surrounding the

6 factors influencing readthrough therapy is awaited.

7

8 Nonsense suppression therapies avoid a number of the issues of alternative gene therapies. As

9 it does not interfere with endogenous gene regulation mechanisms, temporal expression,

10 duration and alternative splicing of target genes remain intact. TRIDs specifically target

11 PTCs, meaning the same compound can work across a variety of genes and diseases. This is

12 particularly convenient for IRDs as the genetic heterogeneity presents a considerable cost-

13 issue per-patient. In addition, small increases of functionally active protein achieved with

14 translational readthrough appear to be of great benefit, particularly in loss-of-function

15 conditions such as choroideremia and Usher syndrome [57,58].

17 However, TRIDs may act on non-pathogenic nonsense variants in other genes and the nearcognate aminoacyl pairing at a PTC may also introduce missense changes, affecting protein activity [59]. This effect is unpredictable but is unlikely to cause additional harm in diseases

20 with no genotype-phenotype correlation such as choroideremia $[15,35,60,61]$. That being

21 said, in some diseases such as aniridia and USH1, missense mutations result in milder disease than null variants so the introduction of missense changes following nonsense suppression

23 may still improve the phenotype $[62,63]$. Nonetheless, the influence of variants must be

24 modelled on a protein-by-protein basis, and failed rescue of phenotype highlights the

25 importance of understanding protein tolerances to amino acid changes [64]. 


\section{Translational readthrough-inducing drugs}

3

4

\subsection{Aminoglycosides}

Aminoglycosides are gram-negative bactericidal antibiotics that contain amino-modified glycosidic linkages. They bind to the adenosine nucleotide in position 1408 in the rRNA sequence of the prokaryotic A-site and induce ribosomal infidelity, disrupted codon recognition, inaccurate mRNA translation and the production of aberrant proteins [65]. A number of aminoglycosides have exhibited readthrough capacity across the decades $[53,66,67]$ but the most effective are gentamicin and G418 [53,67,68] (Fig.3 A-C). These have shown therapeutic potential in both in vitro and in vivo systems for a number of genetic diseases including CF [69] and Duchenne muscular dystrophy (DMD) [70]. However, their clinical use is prevented by considerable renal and oto-toxicity at the doses required for translational readthrough [71], as renal proximal tubule epithelial cells and inner ear hair cells preferentially uptake aminoglycosides [72]. Though this is partially alleviated by coadministration with poly-L-aspartic acid [73], this toxicity prevents its clinical use. In addition, aminoglycosides such as gentamicin are not lipid soluble and have poor penetration through the blood-brain/blood-retinal barrier and the cell membrane [74].

\subsection{Aminoglycoside derivatives}

To enhance readthrough capacity and minimise toxicity, aminoglycoside scaffolds were used to create "designer" aminoglycosides such as the NB compounds $[68,75]$. These exhibit efficient translational readthrough by conservation of the C6'-hydroxyl group, which forms hydrogen bonds with the guanosine nucleotide at position 1408 in eukaryotic rRNA [76] (Fig.3 D-H). Designer aminoglycosides lose antimicrobial properties, suggesting the interaction with prokaryotic rRNA is reduced [75]. Consequently, NB30 treatment confers a 
1 reduced toxicity than traditional aminoglycosides [77,78]. Addition of the (S)-4-amino-2-

2 hydrocybutanoyl (AHB) group, found on the aminoglycoside amikacin (Fig.3 B), to the N1

3 position of NB30 resulted in superior binding with the ribosomal A-site [75,79]. The new

4 compound, NB54 (Fig.3 E), displayed greater in vitro translational readthrough for nonsense

5 mutations in several genes, including USHIC causing type 1 Usher syndrome $[58,80]$.

6 Further modifications resulted in NB74 and NB84, which contained a (R)-6'-methyl group

7 increasing readthrough potency [67] (Fig.3 F,G). Similarly, NB124 (with commercial name

8 ELX-02, Fig.3 H) exhibited a favourable readthrough-toxicity relationship across a number

9 of CF alleles [81]. A phase I clinical trial for ELX-02 for CF showed no adverse effects [82]

10 and a phase II trial has recently been announced for CF patients with the CFTR p.G542*

11 mutation. ELOX-02 has achieved orphan drug status for the treatment of CF and

12 mucopolysaccharidosis type 1 [83].

14 Commercial gentamicin is a mixture of major and minor congeners [84-86], each of which 15 have been subject to readthrough testing. A minor component of gentamicin, X2 (Fig.3 C),

16 has recently showed superior readthrough capacity than gentamicin, NB84 and NB124 and

17 was only beaten in potency by the positive control G418 [87]. However, gentamicin X2

18 presented a superior in vivo safety profile in rodent models compared to G418, hence it is

19 better suited as a therapeutic readthrough agent [87]. However, the historical side effect

20 profile of aminoglycosides and issues with clinical delivery have forced a search for non-

21 aminoglycoside-based small molecule drugs with readthrough capacity.

23 Non-aminoglycoside small molecules with readthrough potential have been discovered from

24 high throughput screening using premature UGA luciferase reporters [88]. PTC124, known as 
1 Ataluren or commercial name Translarna ${ }^{\mathrm{TM}}$ (Fig.3 I), is currently best known to promote 2 readthrough of UGA, UAA and UAG codons, with highest activity for UGA. It also has lower

3 therapeutic concentration ranges than gentamicin, has fewer toxic effects than the classical 4 aminoglycosides, and retains PTC selectivity over natural stop codons [88]. The mechanism of 5 action for PTC124 is still unknown, but it is thought to bind to the ribosome, stimulating 6 sampling of near-cognate tRNAs [89]. It reached phase III clinical trial in cystic fibrosis 7 (NCT02107859) but failed to achieve therapeutic benefit [90]. In a phase III clinical trial for DMD (NCT01826487), none of the children taking the drug lost the ability to walk over the 48 weeks trial period compared with $8 \%$ on the placebo ( 0 out of 47 compared with 4 of 52) [91]. It was predicted to delay loss of walking for up to 7 years and patient experts argued they 11 had seen meaningful stabilisation or improvements in their child's mobility. As a result, PTC124 was approved in the UK for the treatment of DMD. No adverse effects have been noted from the DMD and CF clinical trials [82,90]. It has since been applied to eye diseases with a phase II clinical trial for aniridia currently underway (NTC02647359). Preclinical data in the aniridia mouse model Pax6sey+/-showed reversal of the ocular malformations, improving the retinal histology and responses to light indicating post-natal developmental plasticity

17 (discussed below) [92]. While PTC124 has minimal ocular side effects, good oral bioavailability and is water soluble, concerns still remain regarding penetration through an intact blood-retinal barrier [91].

21 Other small molecule drugs have shown readthrough potential. PTC414 is a derivative of PTC124 and shows comparable readthrough capabilities for all types of PTC in choroideremia in vitro and in vivo models [57]. High-throughput protein transcription/translation enzymelinked immunosorbent (ELISA)-based assays have identified small molecule readthrough (SMRT) compounds with similar three-ring structures to PTC124 such as RTC13 and RTC14 
1 [93] (Fig.3 J,K). RTC13 was shown to restore dystrophin expression and muscle function in

2 mouse models of DMD [94] and efficiencies independent of the position of the PTC within the

3 transcript [95]. GJ071 and GJ072 have similar readthrough efficiency as RTC13 or RTC14 but

4 have greater tolerability based on patient cells with ataxia telangiectasia [93]. Amlexanox

5 (Fig.3 L) is a FDA-approved drug for the treatment of recurrent apthous mouth ulcers [96], but

6 it was recently shown to have dual action of NMD inhibition and nonsense suppression [42].

7 The use of these small molecule compounds on in vitro and in vivo models of IRDs is awaited.

\section{Application in inherited retinal disorders}

10 Several in vitro and in vivo disease models of IRDs have been tested with TRIDs including

11 choroideremia, retinitis pigmentosa, Leber congenital amaurosis and Usher syndrome. A summary of the most relevant studies is listed in Table 1.

14 Choroideremia (CHM) is a X-linked chorioretinal dystrophy affecting 1 in 50,000 to 100,000 males. Features include nyctalopia in childhood, followed by visual field loss during adolescence and progressive blindness by late middle age. The responsible gene is $C H M$, which encodes REP1 (Rab escort protein 1) that mediates vesicular trafficking and posttranslational modification of Rab proteins [15]. Though ubiquitously expressed, systemic manifestations are thought to be mitigated by the presence of the isoform REP2. Phase II and III clinical trials of retinal gene therapy with AAV2 vectors are underway (NCT02407678)

21 [97] but recent reports from Aleman et al report no difference between treated and untreated eyes at year 2 and 3 post-treatment [98]. Over a third of $C H M$ mutations are nonsense variants, making them an appropriate target for nonsense suppression [15]. Zebrafish chmru848 models of CHM carry a UAA PTC and survive for only 5 days post fertilisation due to the 
1 presence of a single REP isoform. Following treatment with gentamicin, paromomycin,

2 PTC124 and PTC414, an increase in survival by up to 2-fold was seen $[57,99]$. Patient

3 fibroblasts with the $C H M$ mutation c. $126 \mathrm{C}>\mathrm{G}, \mathrm{p}$.Y42* were treated with PTC414 and

$4 \quad$ PTC124 and showed recovery of prenylation activity despite no increased protein levels

5 detected [57]. More recently, Torriano et al also investigated the effect of PTC124 on patient

6 fibroblasts and iPSC-RPE carrying a c.772A>T, p.K258* in $C H M$. However, they found no

7 significant trend in functional rescue, despite the addition of NMD and the proteasome

8 inhibitors cycloheximide and MG132 [64]. This failed rescue was attributed to the identity of

9 the missense amino acid that replaced the PTC (tyrosine or glutamine instead of the original

10 lysine), which was determined in silico to be damaging to REP1 function [64]. This

11 exemplifies that a detailed understanding of protein responses to amino acid changes is

12 needed to achieve functional rescue and avoid toxic/gain-of-function side effects. Finally, the

13 application of the NMD inhibitor caffeine to patient $C H M$ fibroblasts resulted in restoration

14 of $C H M$ mRNA to near wild-type levels, suggesting NMD inhibition could be used in

15 conjunction with TRIDs to increase readthrough efficiency [35].

\subsection{Retinitis pigmentosa}

17 Retinitis pigmentosa (RP) is a group of IRDs affecting around 1 in 4000 people [100]. It is characterised by rod photoreceptor cell death causing nyctalopia and visual field loss leading

19 to tunnel vision, followed by cone dysfunction and total blindness. There is significant

20 genetic heterogeneity meaning gene-targeted approaches present a considerable cost per

21 patient issue [101]. The development of effective mutation-dependent approaches, such as

22 nonsense suppression, would be more cost effective and widely applicable.

24 The S334ter rat model of dominant RP has a nonsense variant in the Rho gene creating a 25 gain-of-function Rhodopsin mutant. Rats treated with gentamicin showed reduced abnormal 
1 protein expression, resulting in improvements in retinal histology and vision [53]. In contrast,

2 gentamicin treatment of $r d 12$ transgenic mouse model of RPE65-associated retinopathy did

3 not slow the retinal degeneration [53]. Around 15\% of X-linked RP cases are associated with

4 mutations in the RP2 gene [101-103]. Application of gentamicin to patient lymphoblasts

5 carrying a nonsense mutation of $R P 2$, c. $519 \mathrm{C}>\mathrm{T}, \mathrm{p} . \mathrm{R} 120^{*}$, previously failed to restore full

6 length RP2 protein [102]. However, applying G418 and PTC124 to fibroblasts and iPSC-

7 derived RPE with the same mutation resulted in restoration of $20 \%$ and $13 \%$ of full length

8 functional RP2 protein respectively, as well as improved cellular phenotype [103].

9 Readthrough success due in part to novel TRIDs and a better disease model is a reflection of

10 the advancements in the field over the past 15 years.

12 Deficiency in the MER receptor tyrosine kinase (MERTK) protein in the RPE leads to

13 impaired phagocytosis and a harmful build-up of photoreceptor outer segments (POS),

14 causing RP [104,105]. An AAV2-mediated gene therapy phase I clinical trial

15 (NCT01482195) showed mixed results, with no ophthalmological or systemic toxicity

16 detected at the 2-year follow up but variable clinical response of affected patients included in

17 the study [106]. More recently, patient-derived iPSC-RPE derived from a MERTK compound

18 heterozygous patient, with splice-site (c.61+1G>A) and nonsense (c.1951C>T, p.R651*)

19 variants were treated with PTC124. Full-length functional protein was restored with a $12 \%$

20 increase in phagocytic function [107].

\subsection{Leber congenital amaurosis}

22 Leber congenital amaurosis (LCA) encompasses the severe early onset retinal dystrophies,

23 affecting between 1 in 30,000 to 80,000 people, with 25 causative genes known to date [108].

24 LCA16 is caused by mutations in KCNJ13 [109-111], encoding a potassium channel subunit,

25 Kir7.1, expressed on the RPE surface [112,113]. Shahi et al recently administered NB84 to a 
$1 \quad$ KCNJ13 c.158G>A, p.W56* patient-derived iPSC-RPE model, resulting in restored Kir7.1

2 protein and RPE membrane potential [114]. In vitro and in vivo toxicity studies reported good

3 safety of NB84 [78] meaning translational therapy for LCA16 in animal and human subjects

4 is eagerly awaited.

6 Usher syndrome (USH) is the most common cause of deaf-blindness worldwide, affecting

7 3.2-6.2 per 100,000 people. It is characterised by profound sensorineural hearing loss,

8 variable vestibular dysfunction and RP [115]. Though the hearing loss can be partially

9 improved with hearing aids and cochlear implants, no current treatments exist for the RP

10 [116]. It is clinically and genetically heterogeneous, subdivided into three types (USH1-

11 USH3) according to the clinical severity [117]. The causative genes are expressed in the

12 retinal photoreceptors and stereocilia of the inner ear. A number of USH disease genes such

13 as $U S H 2 A$ and $A D G R V 1$ are too large to package into traditional AAV vectors [118-120],

14 therefore alternative therapies are sought. Nonsense mutations account for $20 \%$ of USH cases

15 [121] (https://www.lovd.nl/). In vitro and ex vivo readthrough of four nonsense mutations in

$16 P C D H 15$ causing USH1 was achieved by Rebibo-Sabbah et al with NB30 and the positive

17 controls gentamicin, G418 and paromomycin [122]. Goldmann et al have displayed

18 readthrough of the $U S H 1 C$ c.91C $>$ T, p.R31* mutation with designer- and non-

19 aminoglycosides NB30, NB54 and PTC124 in in vitro HEK293T cells, ex vivo retinal

20 explants and in vivo mouse models [58]. The most efficient and biocompatible compounds

21 were NB54 and PTC124, and crucially the restored scaffold protein, harmonin, was shown to

22 be functional [58]. Recently, both gentamicin and PTC124 were also shown to increase levels

23 of usherin from reporter constructs containing the mutation c.9424G> T, p.G3142* in USH2A

24 [123]. Furthermore, in patient-derived fibroblasts with the same variant treated with PTC124, 
1 usherin levels increased up to 4.3-fold, and treatment was shown to restore protein membrane

2 localisation and increase cilia formation from $54 \%$ to $78 \%$ of total cells [123].

4 Aniridia, although not an IRD, is a congenital pan-ocular anomaly which has reached the 5 most advanced clinical application with TRIDs. The condition is characterised by iris

6 hypoplasia, cataracts, glaucoma, foveal hypoplasia and corneal anomalies [14]. It affects

7 approximately 1.8 per 100000 live births and presents in isolation or as part of WAGR

8 (Wilms tumour, aniridia, genitourinary anomalies and intellectual disability) syndrome

$9 \quad[63,124]$. Non-syndromic aniridia is mostly associated with heterozygous mutations in PAX6

10 leading to haploinsufficiency of this transcription factor, which has a pivotal role in ocular

11 development [14]. Nearly half of patients with PAX6 mutations carry nonsense variants

$12[14,125]$, making this disease an ideal target for nonsense suppression approaches. The

13 Pax6sey+/- small eye mouse model of aniridia contains a c.580G>T, p.G194* PTC and

14 typically presents with a thickened cornea, underdeveloped lens as well as a thickened and

15 infolded retina [126]. Systemic treatment of Pax6sey+/- with PTC124 (and gentamicin)

16 increased lens size, improved retinal infolding and photoreceptor density but had limited

17 effect on the cornea thickening [92]. In fact, corneal improvement was best achieved with

18 topical administration of PTC124, which restored PAX6 to 90\% of wildtype levels in both

19 cornea and retina of affected animals [92,127]. A phase II clinical trial for the treatment of

20 aniridia with oral suspension of PTC124 (TranslarnatM) is underway (NCT02647359).

\section{Current limitations of translational readthrough-inducing drugs}

23 The availability of PTC-containing transcripts is what most directly limits the efficiency of current nonsense suppression approaches. This availability is dependent on the NMD process 
1 as outlined previously. Attempts to better understand NMD regulators must account for

2 species-specific differences in genomic context and epigenetic influences, both of which

3 must be better understood [32,128]. For example, the human $C H M$ gene contains an open

4 reading frame (ORF) between -20 of the main AUG and a termination codon at +32 . These

5 are initiation codons in the 5'UTR that are out of frame to the main coding sequence. By

6 helping identify termination codons as premature they trigger NMD [129]. The zebrafish

7 ortholog chm lacks an upstream ORF, meaning NMD may be less effective in this model

8 compared to humans. Differences like this may change the response to translational

9 readthrough seen. For example, PTC124 reportedly does not affect NMD [88,103,130],

10 however Moosajee et al found increased levels of mRNA following dosing of chmrus48

11 zebrafish with PTC124 suggesting reduced NMD efficiency [57]. There is therefore a need to

12 understand species-specific NMD and nonsense suppression mechanisms. The identity of the

13 substituted amino acid is also important to target protein function. This must be modelled on

14 an individual basis in preclinical models, even in those where missense mutations derive

15 milder disease phenotype [62,125].

17 Methods of TRID delivery to the retina requires further development. Though the blood

18 ocular barrier is disrupted in many retinal dystrophies, its presence reduces systemic drug

19 absorption of TRIDs into the retina (though this is molecule dependent) [74]. Fortunately, the

20 retina can be approached with local administration, either topical or intraocular. This

21 administration would reduce systemic side effects, allowing for higher therapeutic doses to be

22 delivered to the eye. The START formulation is a topical Ataluren mixture designed to

23 effectively deliver Ataluren as a topical therapy without ocular irritation. The formulation of

$24 \quad 0.9 \%$ Sodium chloride, $1 \%$ Tween $80,1 \%$ powdered Ataluren, and $1 \%$ 
1 carboxymethylcellulose was found to maximise particle dispersion and increase suspension

2 viscosity. It had much reduced ocular irritation compared with a $1 \%$ aqueous Ataluren

3 suspension [92]. As described in section 6.5, treatment of the Pax6sey+/- mouse with topical

4 PTC124 using the START formulation restored PAX6 levels to 90\% of wild-type, which was

5 not achieved by systemic administration $[92,127]$. The penetration of topical PTC124 to the

6 retina suggests that treatment of retinal dystrophies with similar non-invasive deliveries is an

7 exciting possibility. The current aniridia trial is with oral PTC124 (TranslarnaTM) suspension

8 but further clinical trials of local TRID administration (topical and intraocular) are awaited

10 Another approach to improve localised delivery and cell membrane uptake of TRIDs is their

11 encapsulation within a liposome [131]. The slower release of these formulations was thought

12 to reduce PTC suppression as aminoglycosides deliver peak-driven readthrough capacity

13 [132]. Injecting gentamicin within lysosomes into a mouse model of DMD found increased

14 readthrough compared to gentamicin alone [133]. Finally, to allow for sustained drug

15 delivery to the retina, nonsense suppression therapies may in the future utilise delivery

16 devices such as nanoporous film devices, which have been developed for use in more

17 common retinal diseases such as diabetic retinopathy or age-related macular degeneration

$18[134,135]$.

19

\section{Conclusion}

21 Nonsense suppression therapy is a method of pharmacological gene therapy that could help millions of patients across the globe. IRDs are a prime target for nonsense suppression due to

23 their preponderance for nonsense mutations and the devastating blindness they currently

24 cause. This review highlights the exciting preclinical promise of nonsense suppression

25 therapy for the treatment of IRDs. Though Translarnatm has reached clinical trial 
1 (NCT02647359) for aniridia, the preclinical developments described create anticipation for

2 more clinical trials, including for other compounds.

\section{Expert's Opinion}

4 This review presents extensive evidence supporting TRIDs as a promising therapeutic approach for the treatment of genetic eye disorders caused by nonsense mutations, with special focus on IRDs. Over the next five years, we expect to see the translation of preclinical data into clinical trials. Current limitations of TRIDs such as readthrough efficiency, toxicity and delivery methods are also expected to improve, helped by the development of novel

9 TRIDs alongside more sensitive detection and formulations in representative human disease models.

Advances in next generation sequencing, such as whole genome sequencing, are increasing our genetic diagnostic rates for patients. Using information such as PTC identity and genomic context in patient-specific in vitro models may predict response to nonsense suppression therapy or provide indicators as to which small molecule drug may be more suited for the individual, as a form of personalised medicine. Patient derived iPSC-RPE or 3D retinal organoids are an accurate model of human disease, which provide valuable insights on diseases mechanisms and can be powerful platforms for drug development. In fact, patientspecific retinal organoids have been used as proof-of-concept models for other therapeutics including CRISPR/Cas9 gene correction, antisense oligonucleotides and AAV delivery [136$138]$.

Preclinical studies must also pay close attention to study endpoints. Some studies of TRIDs have demonstrated functional benefits without a detectable increase in protein levels in several models, such as increased rab prenylation in human nonsense-mediated 
1 choroideremia fibroblasts with PTC124 [57] and reduced glycosaminoglycan accumulation in

2 the Idua MPS I-H mouse model with NB84 [139]. This may be due to reduced sensitivity of

3 existing antibodies, hence functional endpoints/assays are important.

5 The exact mode of action for some TRIDs, such as PTC124, remains unknown. Further work

6 is required to determine this and may provide an explanation of why some drugs work better

7 on PTCs. Another factor to consider when improving TRIDs efficiency is NMD. Better

8 understanding of why there appears to be varied NMD efficiency across patients with the

9 same variant, between patients with mutations in the same gene, and even across different

10 tissues within the same patient is required. Recent evidence points to the use of NMD

11 inhibitors, such as caffeine, in parallel with TRIDs to further improve readthrough efficiency

$12[39,41]$. Further developments on this strategy or even the identification of more compounds

13 with double action, like amlexanox, are expected and their progress into clinical trials for

14 IRDs is eagerly awaited.

16 The systemic administration of both TRIDs and NMD inhibitors may increase the number of 17 harmful truncated proteins and off-target effects. Local delivery to the eye may be safer for patients. Topical formulations have already been shown to penetrate to the posterior segment and may become the administration method of choice for IRDs, perhaps aided by

20 nanotechnology-based developments $[134,140]$. If both eyes are being treated

21 simultaneously, we require a detailed understanding of the natural history of each IRD, with reliable biomarkers and outcome metrics to assay the response to drug treatment.

24 In conclusion, patient pre-screening may be required to determine the optimal nonsense

25 suppression therapy for best prognostic outcome dependent on PTC, genomic context, 
1 baseline transcript levels/NMD activity. Localised administration to the eyes would further

2 maximise the therapeutic effect in IRD patients, by increasing drug availability and reducing

3 off target effects.

\section{$5 \quad$ References}

[1] Liew G, Michaelides M, Bunce C. A comparison of the causes of blindness certifications in England and Wales in working age adults (16-64 years), 1999-2000 with 2009-2010. BMJ Open. 2014;4:e004015.

[2] Frick KD, Roebuck MC, Feldstein JI, et al. Health services utilization and cost of retinitis pigmentosa. Arch. Ophthalmol. (Chicago, III. 1960). 2012;130:629-634.

[3] Hamblion EL, Moore AT, Rahi JS. The health-related quality of life of children with hereditary retinal disorders and the psychosocial impact on their families. Investig. Ophthalmol. Vis. Sci. 2011;52:7981-7986.

[4] Pierce EA, Bennett J. The Status of RPE65 Gene Therapy Trials: Safety and Efficacy. Cold Spring Harb. Perspect. Med. 2015;5:a017285-a017285.

[5] Cideciyan A V., Jacobson SG, Beltran WA, et al. Human retinal gene therapy for Leber congenital amaurosis shows advancing retinal degeneration despite enduring visual improvement. Proc. Natl. Acad. Sci. 2013;110:E517-E525.

[6] MacLaren RE, Groppe M, Barnard AR, et al. Retinal gene therapy in patients with choroideremia: initial findings from a phase 1/2 clinical trial. Lancet. 2014;383:1129-1137.

[7] Petit L, Khanna H, Punzo C. Advances in Gene Therapy for Diseases of the Eye. Hum. Gene Ther. 2016;27:563-579.

[8] Dias N, Stein CA. Antisense oligonucleotides: basic concepts and mechanisms. Mol. Cancer Ther. 2002;1:347-355.

[9] Dulla K, Aguila M, Lane A, et al. Splice-Modulating Oligonucleotide QR-110 Restores CEP290 mRNA and Function in Human c.2991+1655A>G LCA10 Models. Mol. Ther. - Nucleic Acids. 2018;12:730-740.

[10] Slijkerman RW, Vaché C, Dona M, et al. Antisense Oligonucleotide-based Splice Correction for USH2A-associated Retinal Degeneration Caused by a Frequent Deep-intronic Mutation. Mol. Ther. Nucleic Acids. 2016;5:e381.

[11] Collin RW, den Hollander Al, van der Velde-Visser SD, et al. Antisense Oligonucleotide (AON)-based Therapy for Leber Congenital Amaurosis Caused by a Frequent Mutation in CEP290. Mol. Ther. - Nucleic Acids. 2012;1:e14.

[12] Zanardi TA, Kim T-W, Shen L, et al. Chronic Toxicity Assessment of 2'- O -Methoxyethyl Antisense Oligonucleotides in Mice . Nucleic Acid Ther. 2018;28:233-241.

[13] Mort M, Ivanov D, Cooper DN, et al. A meta-analysis of nonsense mutations causing human genetic disease. Hum. Mutat. 2008;29:1037-1047.

[14] Richardson R, Hingorani M, Van Heyningen V, et al. Clinical utility gene card for: Aniridia. Eur. J. Hum. Genet. 2016;24:4-4.

[15] Moosajee M, Ramsden SC, Black GC, et al. Clinical utility gene card for: Choroideremia. Eur. J. Hum. Genet. 2014;22:572-572.

[16] Mendell JT, Dietz HC. When the message goes awry: disease-producing mutations that influence mRNA content and performance. Cell. 2001;107:411-414.

[17] Lewis BP, Green RE, Brenner SE. Evidence for the widespread coupling of alternative splicing and nonsensemediated mRNA decay in humans. Proc. Natl. Acad. Sci. 2003;100:189-192.

[18] Pan Q, Saltzman AL, Kim YK, et al. Quantitative microarray profiling provides evidence against widespread coupling of alternative splicing with nonsense-mediated mRNA decay to control gene expression. Genes Dev. 2006;20:153158.

[19] Bidou L, Allamand V, Rousset J-P, et al. Sense from nonsense: therapies for premature stop codon diseases. Trends Mol. Med. 2012;18:679-688.

[20] Inoue K, Khajavi M, Ohyama T, et al. Molecular mechanism for distinct neurological phenotypes conveyed by allelic truncating mutations. Nat. Genet. 2004;36:361-369.

[21] Manuvakhova M, Keeling K, Bedwell DM. Aminoglycoside antibiotics mediate context-dependent suppression of termination codons in a mammalian translation system. RNA. 2000;6:1044-1055.

[22] Kervestin S, Jacobson A. NMD: a multifaceted response to premature translational termination. Nat. Rev. Mol. Cell Biol. 2012;13:700-712.

[23] Celik A, Kervestin S, Jacobson A. NMD: At the crossroads between translation termination and ribosome recycling. Biochimie. 2015;114:2-9.

[24] Yamashita A, Izumi N, Kashima I, et al. SMG-8 and SMG-9, two novel subunits of the SMG-1 complex, regulate remodeling of the mRNA surveillance complex during nonsense-mediated mRNA decay. Genes Dev. 
2009;23:1091-1105.

[25] Ivanov P V, Gehring NH, Kunz JB, et al. Interactions between UPF1, eRFs, PABP and the exon junction complex suggest an integrated model for mammalian NMD pathways. EMBO J. 2008;27:736-747.

[26] He F, Jacobson A. Nonsense-Mediated mRNA Decay: Degradation of Defective Transcripts Is Only Part of the Story. Annu. Rev. Genet. 2015;49:339-366.

[27] Lykke-Andersen J, Shu M Di, Steitz JA. Human Upf proteins target an mRNA for nonsense-mediated decay when downstream of a termination codon. Cell. 2000;103:1121-1131.

[28] He F, Li X, Spatrick P, et al. Genome-Wide Analysis of mRNAs Regulated by the Nonsense-Mediated and $5^{\prime}$ to $3^{\prime}$ mRNA Decay Pathways in Yeast. Mol. Cell. 2003;12:1439-1452.

[29] Silva AL, Ribeiro $P$, Inacio A, et al. Proximity of the poly(A)-binding protein to a premature termination codon inhibits mammalian nonsense-mediated mRNA decay. RNA. 2008;14:563-576.

[30] Pereira FJC, Teixeira A, Kong J, et al. Resistance of mRNAs with AUG-proximal nonsense mutations to nonsensemediated decay reflects variables of mRNA structure and translational activity. Nucleic Acids Res. 2015;43:65286544.

[31] Barbosa C, Peixeiro I, Romão L. Gene Expression Regulation by Upstream Open Reading Frames and Human Disease. Fisher EMC, editor. PLoS Genet. 2013;9:e1003529.

[32] Zetoune $A B$, Fontanière $S$, Magnin $D$, et al. Comparison of nonsense-mediated mRNA decay efficiency in various murine tissues. BMC Genet. 2008;9:83.

[33] Linde L, Boelz S, Nissim-Rafinia M, et al. Nonsense-mediated mRNA decay affects nonsense transcript levels and governs response of cystic fibrosis patients to gentamicin. J. Clin. Invest. 2007;117:683-692.

[34] Nguyen LS, Wilkinson MF, Gecz J. Nonsense-mediated mRNA decay: Inter-individual variability and human disease. Neurosci. Biobehav. Rev. 2014;46:175-186.

[35] Sarkar H, Mitsios A, Smart M, et al. Nonsense-mediated mRNA decay efficiency varies in choroideremia providing a target to boost small molecule therapeutics. Hum. Mol. Genet. 2019;28:1865-1871.

[36] Yamashita A, Ohnishi T, Kashima I, et al. Human SMG-1, a novel phosphatidylinositol 3-kinase-related protein kinase, associates with components of the mRNA surveillance complex and is involved in the regulation of nonsense-mediated mRNA decay. Genes Dev. 2001;15:2215-2228.

[37] Usuki F, Yamashita A, Higuchi I, et al. Inhibition of nonsense-mediated mRNA decay rescues the phenotype in Ullrich's disease. Ann. Neurol. 2004;55:740-744.

[38] Durand S, Cougot N, Mahuteau-Betzer F, et al. Inhibition of nonsense-mediated mRNA decay (NMD) by a new chemical molecule reveals the dynamic of NMD factors in P-bodies. J. Cell Biol. 2007;178:1145-1160.

[39] Keeling KM, Wang D, Dai Y, et al. Attenuation of Nonsense-Mediated mRNA Decay Enhances In Vivo Nonsense Suppression. Reiner DJ, editor. PLoS One. 2013;8:e60478.

[40] Gotham VJB, Hobbs MC, Burgin R, et al. Synthesis and activity of a novel inhibitor of nonsense-mediated mRNA decay. Org. Biomol. Chem. 2016;14:1559-1563.

[41] Atanasova VS, Jiang Q, Prisco M, et al. Amlexanox Enhances Premature Termination Codon Read-Through in COL7A1 and Expression of Full Length Type VII Collagen: Potential Therapy for Recessive Dystrophic Epidermolysis Bullosa. J. Invest. Dermatol. 2017;137:1842-1849.

[42] Gonzalez-Hilarion S, Beghyn T, Jia J, et al. Rescue of nonsense mutations by amlexanox in human cells. Orphanet J. Rare Dis. 2012;7:58.

[43] Seoighe C, Gehring C. Heritability in the efficiency of nonsense-mediated mRNA decay in humans. PLoS One. 2010;5:e11657.

[44] Rodnina M V., Gromadski KB, Kothe U, et al. Recognition and selection of tRNA in translation. FEBS Lett. 2005;579:938-942.

[45] Keeling KM, Wang D, Conard SE, et al. Suppression of premature termination codons as a therapeutic approach. Crit. Rev. Biochem. Mol. Biol. 2012;47:444-463.

[46] Richardson R, Smart M, Tracey-White D, et al. Mechanism and evidence of nonsense suppression therapy for genetic eye disorders. Exp. Eye Res. 2017;155:24-37.

[47] Dabrowski M, Bukowy-Bieryllo Z, Zietkiewicz E. Translational readthrough potential of natural termination codons in eucaryotes - The impact of RNA sequence. RNA Biol. 2015;12:950-958.

[48] Floquet C, Hatin I, Rousset J-P, et al. Statistical Analysis of Readthrough Levels for Nonsense Mutations in Mammalian Cells Reveals a Major Determinant of Response to Gentamicin. Flanigan KM, editor. PLoS Genet. 2012;8:e1002608.

[49] Keeling KM, Xue X, Gunn G, et al. Therapeutics based on stop codon readthrough. Annu. Rev. Genomics Hum. Genet. 2014/04/18. 2014;15:371-394.

[50] Howard MT, Shirts BH, Petros LM, et al. Sequence specificity of aminoglycoside-induced stop condon readthrough: potential implications for treatment of Duchenne muscular dystrophy. Ann. Neurol. 2000;48:164-169.

[51] Bonetti B, Fu L, Moon J, et al. The efficiency of translation termination is determined by a synergistic interplay between upstream and downstream sequences in Saccharomyces cerevisiae. J. Mol. Biol. 1995;251:334-345.

[52] Tork S, Hatin I, Rousset JP, et al. The major 5' determinant in stop codon read-through involves two adjacent adenines. Nucleic Acids Res. 2004;32:415-421.

[53] Guerin K, Gregory-Evans CY, Hodges MD, et al. Systemic aminoglycoside treatment in rodent models of retinitis pigmentosa. Exp. Eye Res. 2008;87:197-207. 
[54] Carnes J, Jacobson M, Leinwand L, et al. Stop codon suppression via inhibition of eRF1 expression. RNA. 2003;9:648-653.

[55] Singleton RS, Liu-Yi P, Formenti F, et al. OGFOD1 catalyzes prolyl hydroxylation of RPS23 and is involved in translation control and stress granule formation. Proc. Natl. Acad. Sci. U. S. A. 2014;111:4031-4036.

[56] Loenarz C, Sekirnik R, Thalhammer A, et al. Hydroxylation of the eukaryotic ribosomal decoding center affects translational accuracy. Proc. Natl. Acad. Sci. U. S. A. 2014;111:4019-4024.

[57] Moosajee M, Tracey-White D, Smart M, et al. Functional rescue of REP1 following treatment with PTC124 and novel derivative PTC-414 in human choroideremia fibroblasts and the nonsense-mediated zebrafish model. Hum. Mol. Genet. 2016;25:3416-3431.

[58] Goldmann T, Overlack N, Möller F, et al. A comparative evaluation of NB30, NB54 and PTC124 in translational read-through efficacy for treatment of an USH1C nonsense mutation. EMBO Mol. Med. 2012;4:1186-1199.

[59] Bordeira-Carriço R, Pêgo AP, Santos M, et al. Cancer syndromes and therapy by stop-codon readthrough. Trends Mol. Med. 2012;18:667-678.

[60] Sergeev YV, Smaoui N, Sui R, et al. The functional effect of pathogenic mutations in Rab escort protein 1. Mutat. Res. Mol. Mech. Mutagen. 2009;665:44-50.

[61] Esposito G, De Falco F, Tinto N, et al. Comprehensive mutation analysis (20 families) of the choroideremia gene reveals a missense variant that prevents the binding of REP1 with rab geranylgeranyl transferase. Hum. Mutat. 2011;32:1460-1469.

[62] Ouyang X, Xia X, Verpy E, et al. Mutations in the alternatively spliced exons of USH1C cause non-syndromic recessive deafness. Hum. Genet. 2002;111:26-30.

[63] Lima Cunha D, Arno G, Corton M, et al. The Spectrum of PAX6 Mutations and Genotype-Phenotype Correlations in the Eye. Genes (Basel). 2019;10.

[64] Torriano S, Erkilic N, Baux D, et al. The effect of PTC124 on choroideremia fibroblasts and iPSC-derived RPE raises considerations for therapy. Sci. Rep. 2018;8:8234.

[65] Mingeot-Leclercq MP, Glupczynski Y, Tulkens PM. Aminoglycosides: activity and resistance. Antimicrob. Agents Chemother. 1999;43:727-737.

[66] Nagel-Wolfrum K, Möller F, Penner I, et al. Targeting Nonsense Mutations in Diseases with Translational ReadThrough-Inducing Drugs (TRIDs). BioDrugs. 2016;30:49-74.

[67] Nudelman I, Glikin D, Smolkin B, et al. Repairing faulty genes by aminoglycosides: Development of new derivatives of geneticin (G418) with enhanced suppression of diseases-causing nonsense mutations. Bioorg. Med. Chem. 2010;18:3735-3746.

[68] Hainrichson M, Nudelman I, Baasov T. Designer aminoglycosides: the race to develop improved antibiotics and compounds for the treatment of human genetic diseases. Org. Biomol. Chem. 2008;6:227-239.

[69] Du M, Jones JR, Lanier J, et al. Aminoglycoside suppression of a premature stop mutation in a Cftr-/- mouse carrying a human CFTR-G542X transgene. J. Mol. Med. 2002;80:595-604.

[70] Shoturma DI, Leland SE, Cordier L, et al. Aminoglycoside antibiotics restore dystrophin function to skeletal muscles of mdx mice. J. Clin. Invest. 2008;104:375-381.

[71] Floquet C, Rousset J-P, Bidou L. Readthrough of Premature Termination Codons in the Adenomatous Polyposis Coli Gene Restores Its Biological Activity in Human Cancer Cells. Deb S, editor. PLoS One. 2011;6:e24125.

[72] Moestrup SK, Cui S, Vorum H, et al. Evidence that epithelial glycoprotein 330/megalin mediates uptake of polybasic drugs. J. Clin. Invest. 1995;96:1404-1413.

[73] Du M, Keeling KM, Fan L, et al. Poly-L-aspartic acid enhances and prolongs gentamicin-mediated suppression of the CFTR-G542X mutation in a cystic fibrosis mouse model. J. Biol. Chem. 2009;284:6885-6892.

[74] Fiscella RG, Gieser J, Phillpotts B, et al. Intraocular penetration of gentamicin after once-daily aminoglycoside dosing. Retina. 1998;18:339-342.

[75] Nudelman I, Rebibo-Sabbah A, Shallom-Shezifi D, et al. Redesign of aminoglycosides for treatment of human genetic diseases caused by premature stop mutations. Bioorg. Med. Chem. Lett. 2006;16:6310-6315.

[76] Pfister P, Hobbie S, Vicens Q, et al. The Molecular Basis for A-Site Mutations Conferring Aminoglycoside Resistance: Relationship between Ribosomal Susceptibility and X-ray Crystal Structures. ChemBioChem. 2003;4:1078-1088.

[77] Goldmann T, Rebibo-Sabbah A, Overlack N, et al. Beneficial read-through of a USH1C nonsense mutation by designed aminoglycoside NB30 in the retina. Investig. Ophthalmol. Vis. Sci. 2010;51:6671-6680.

[78] Shulman E, Belakhov V, Wei G, et al. Designer aminoglycosides that selectively inhibit cytoplasmic rather than mitochondrial ribosomes show decreased ototoxicity: a strategy for the treatment of genetic diseases. J. Biol. Chem. 2014;289:2318-2330.

[79] Kondo J, Hainrichson M, Nudelman I, et al. Differential Selectivity of Natural and Synthetic Aminoglycosides towards the Eukaryotic and Prokaryotic Decoding A Sites. ChemBioChem. 2007;8:1700-1709.

[80] Nudelman I, Rebibo-Sabbah A, Cherniavsky M, et al. Development of Novel Aminoglycoside (NB54) with Reduced Toxicity and Enhanced Suppression of Disease-Causing Premature Stop Mutations. J. Med. Chem. 2009;52:28362845.

[81] Xue X, Mutyam V, Tang L, et al. Synthetic Aminoglycosides Efficiently Suppress Cystic Fibrosis Transmembrane Conductance Regulator Nonsense Mutations and Are Enhanced by Ivacaftor. Am. J. Respir. Cell Mol. Biol. 2014;50:805. 
[82] Leubitz A, Frydman-Marom A, Sharpe N, et al. Safety, Tolerability, and Pharmacokinetics of Single Ascending Doses of ELX-02, a Potential Treatment for Genetic Disorders Caused by Nonsense Mutations, in Healthy Volunteers. Clin. Pharmacol. Drug Dev. 2019;

[83] Orphanet. 6'-(R)-methyl-5-O-(5-amino-5,6-dideoxy-alpha-L-talofuranosyl)-par omamine sulfate [Internet]. Orphanet an online database rare Dis. orphan drugs. Copyright, Inser. 1997. 1997 [cited 2019 Oct 21]. Available from: https://www.orpha.net/consor/cgi-bin/Drugs_Search.php?lng=EN\&data_id=3265\&Substance=6---R-methyl-5-0--5-amino-5-6-dideoxy-alpha-L-talofuranosyl--paromamine-

sulfate\&search=Drugs_Search_SubstanceTradename\&data_type=Product\&diseaseType=Drug\&Typ=Sub\&title=\&d.

[84] Deubner R, Schollmayer C, Wienen F, et al. Assignment of the major and minor components of gentamicin for evaluation of batches. Magn. Reson. Chem. 2003;41:589-598.

[85] Vydrin AF, Shikhaleev I V., Makhortov VL, et al. Component Composition of Gentamicin Sulfate Preparations. Pharm. Chem. J. 2003;37:448-450.

[86] Stypulkowska K, Blazewicz A, Fijalek Z, et al. Determination of Gentamicin Sulphate Composition and Related Substances in Pharmaceutical Preparations by LC with Charged Aerosol Detection. Chromatographia. 2010;72:1225-1229.

[87] Friesen WJ, Johnson B, Sierra J, et al. The minor gentamicin complex component, $\mathrm{X} 2$, is a potent premature stop codon readthrough molecule with therapeutic potential. PLoS One. 2018;13:e0206158.

[88] Welch EM, Barton ER, Zhuo J, et al. PTC124 targets genetic disorders caused by nonsense mutations. Nature. 2007;447:87-91.

[89] Roy B, Friesen WJ, Tomizawa Y, et al. Ataluren stimulates ribosomal selection of near-cognate tRNAs to promote nonsense suppression. Proc. Natl. Acad. Sci. U. S. A. 2016;113:12508-12513.

[90] Kerem E, Konstan MW, De Boeck K, et al. Ataluren for the treatment of nonsense-mutation cystic fibrosis: a randomised, double-blind, placebo-controlled phase 3 trial. Lancet Respir. Med. 2014;2:539-547.

[91] McDonald CM, Campbell C, Torricelli RE, et al. Ataluren in patients with nonsense mutation Duchenne muscular dystrophy (ACT DMD): a multicentre, randomised, double-blind, placebo-controlled, phase 3 trial. Lancet. 2017;390:1489-1498.

[92] Gregory-Evans CY, Wang X, Wasan KM, et al. Postnatal manipulation of Pax6 dosage reverses congenital tissue malformation defects. J. Clin. Invest. 2014;124:111-116.

[93] Du L, Jung ME, Damoiseaux R, et al. A new series of small molecular weight compounds induce read through of all three types of nonsense mutations in the ATM gene. Mol. Ther. 2013;21:1653-1660.

[94] Kayali R, Ku J-M, Khitrov G, et al. Read-through compound 13 restores dystrophin expression and improves muscle function in the mdx mouse model for Duchenne muscular dystrophy. Hum. Mol. Genet. 2012;21:4007-4020.

[95] Du L, Damoiseaux R, Nahas S, et al. Nonaminoglycoside compounds induce readthrough of nonsense mutations. J. Exp. Med. 2009;206:2285-2297.

[96] Bell J. Amlexanox for the treatment of recurrent aphthous ulcers. Clin. Drug Investig. 2005;25:555-566.

[97] Xue K, MacLaren RE. Ocular gene therapy for choroideremia: clinical trials and future perspectives. Expert Rev. Ophthalmol. 2018;13:129-138.

[98] Aleman TS, Huckfeldt RM, Serrano L, et al. AAV2-hCHM Subretinal Delivery to the Macula in Choroideremia: 2 year Results of an Ongoing Phase I/II Gene Therapy Trial. Invest Ophthalmol Vis Sci. 2019;60:5173.

[99] Moosajee M, Gregory-Evans K, Ellis CD, et al. Translational bypass of nonsense mutations in zebrafish rep1, pax2.1 and lamb1 highlights a viable therapeutic option for untreatable genetic eye disease. Hum. Mol. Genet. 2008;17:3987-4000.

[100] Hamel C. Retinitis pigmentosa. Orphanet J. Rare Dis. 2006;1:40.

[101] Dryja TP, McGee TL, Hahn LB, et al. Mutations within the Rhodopsin Gene in Patients with Autosomal Dominant Retinitis Pigmentosa. N. Engl. J. Med. 1990;323:1302-1307.

[102] Grayson C, Chapple JP, Willison KR, et al. In vitro analysis of aminoglycoside therapy for the Arg120stop nonsense mutation in RP2 patients. J. Med. Genet. 2002;39:62-67.

[103] Schwarz N, Carr A-J, Lane A, et al. Translational read-through of the RP2 Arg120stop mutation in patient iPSCderived retinal pigment epithelium cells. Hum. Mol. Genet. 2015;24:972-986.

[104] Ostergaard E, Duno M, Batbayli M, et al. A novel MERTK deletion is a common founder mutation in the Faroe Islands and is responsible for a high proportion of retinitis pigmentosa cases. Mol. Vis. 2011;17:1485-1492.

[105] Feng W, Yasumura D, Matthes MT, et al. Mertk Triggers Uptake of Photoreceptor Outer Segments during Phagocytosis by Cultured Retinal Pigment Epithelial Cells. J. Biol. Chem. 2002;277:17016-17022.

[106] Ghazi NG, Abboud EB, Nowilaty SR, et al. Treatment of retinitis pigmentosa due to MERTK mutations by ocular subretinal injection of adeno-associated virus gene vector: results of a phase I trial. Hum. Genet. 2016;135:327343.

[107] Ramsden CM, Nommiste B, R. Lane A, et al. Rescue of the MERTK phagocytic defect in a human iPSC disease model using translational read-through inducing drugs. Sci. Rep. 2017;7:51.

[108] Kumaran N, Moore AT, Weleber RG, et al. Leber congenital amaurosis/early-onset severe retinal dystrophy: Clinical features, molecular genetics and therapeutic interventions. Br. J. Ophthalmol. BMJ Publishing Group; 2017. p. 1147-1154.

[109] Pattnaik BR, Shahi PK, Marino MJ, et al. A Novel KCNJ13 Nonsense Mutation and Loss of Kir7.1 Channel Function Causes Leber Congenital Amaurosis (LCA16). Hum. Mutat. 2015;36:720-727. 
[110] Sergouniotis PI, Davidson AE, Mackay DS, et al. Recessive Mutations in KCNJ13, Encoding an Inwardly Rectifying Potassium Channel Subunit, Cause Leber Congenital Amaurosis. Am. J. Hum. Genet. 2011;89:183-190.

[111] Roman D, Zhong H, Yaklichkin S, et al. Conditional loss of Kcnj13 in the retinal pigment epithelium causes photoreceptor degeneration. Exp. Eye Res. 2018;176:219-226.

[112] Kumar M, Pattnaik BR. Focus on Kir7.1: Physiology and channelopathy. Channels. 2015;8:488-495.

[113] la Cour M. The retinal pigment epithelium controls the potassium activity in the subretinal space. Acta Ophthalmol. Suppl. (Oxf. ). 1985;173:9-10.

[114] Shahi PK, Hermans D, Sinha D, et al. Gene Augmentation and Readthrough Rescue Channelopathy in an iPSC-RPE Model of Congenital Blindness. Am. J. Hum. Genet. 2019;104:310-318.

[115] Toms M, Bitner-Glindzicz M, Webster A, et al. Usher syndrome: a review of the clinical phenotype, genes and therapeutic strategies. Expert Rev. Ophthalmol. 2015;10:241-256.

[116] Damen GWJA, Beynon AJ, Krabbe PFM, et al. Cochlear implantation and quality of life in postlingually deaf adults: Long-term follow-up. Otolaryngol. Neck Surg. 2007;136:597-604.

[117] Saihan Z, Webster AR, Luxon L, et al. Update on Usher syndrome. Curr. Opin. Neurol. 2009;22:19-27.

[118] Nagel-Wolfrum K, Baasov T, Wolfrum U. Therapy Strategies for Usher Syndrome Type 1C in the Retina. Springer, New York, NY; 2014. p. 741-747.

[119] Reiners J, Nagel-Wolfrum K, Jürgens K, et al. Molecular basis of human Usher syndrome: Deciphering the meshes of the Usher protein network provides insights into the pathomechanisms of the Usher disease. Exp. Eye Res. 2006;83:97-119.

[120] Neuhaus C, Eisenberger T, Decker C, et al. Next-generation sequencing reveals the mutational landscape of clinically diagnosed Usher syndrome: copy number variations, phenocopies, a predominant target for translational read-through, and PEX26 mutated in Heimler syndrome. Mol. Genet. Genomic Med. 2017;5:531-552.

[121] Claustres M. Molecular genetic analysis of rare diseases in 2007 : selected examples 2007. Research Signpost; 2007.

[122] Rebibo-Sabbah A, Nudelman I, Ahmed ZM, et al. In vitro and ex vivo suppression by aminoglycosides of PCDH15 nonsense mutations underlying type 1 Usher syndrome. Hum. Genet. 2007;122:373-381.

[123] Samanta A, Stingl K, Kohl S, et al. Ataluren for the Treatment of Usher Syndrome 2A Caused by Nonsense Mutations. Int. J. Mol. Sci. 2019;20.

[124] Berlin HS, Ritch R. The treatment of glaucoma secondary aniridia. Mt. Sinai J. Med. 1981;48:111-115.

[125] Hingorani M, Williamson KA, Moore AT, et al. Detailed ophthalmologic evaluation of 43 individuals with PAX6 mutations. Investig. Ophthalmol. Vis. Sci. 2009;50:2581-2590.

[126] Hill RE, Favor J, Hogan BLM, et al. Mouse Small eye results from mutations in a paired-like homeobox-containing gene. Nature. 1991;354:522-525.

[127] Wang X, Gregory-Evans K, Wasan KM, et al. Efficacy of Postnatal In Vivo Nonsense Suppression Therapy in a Pax6 Mouse Model of Aniridia. Mol. Ther. Nucleic Acids. 2017;7:417-428.

[128] Thada V, Miller JN, Kovács AD, et al. Tissue-specific variation in nonsense mutant transcript level and drug-induced read-through efficiency in the Cln1 ${ }^{\text {R151X }}$ mouse model of INCL. J. Cell. Mol. Med. 2016;20:381-385.

[129] Calvo SE, Pagliarini DJ, Mootha VK. Upstream open reading frames cause widespread reduction of protein expression and are polymorphic among humans. Proc. Natl. Acad. Sci. 2009;106:7507-7512.

[130] Du M, Liu X, Welch EM, et al. PTC124 is an orally bioavailable compound that promotes suppression of the human CFTR-G542X nonsense allele in a CF mouse model. Proc. Natl. Acad. Sci. 2008;105:2064-2069.

[131] Schiffelers R. Liposome-encapsulated aminoglycosides in pre-clinical and clinical studies. J. Antimicrob. Chemother. 2001;48:333-344.

[132] Barton-Davis ER, Cordier L, Shoturma DI, et al. Aminoglycoside antibiotics restore dystrophin function to skeletal muscles of mdx mice. J. Clin. Invest. 1999;104:375-381.

[133] Yukihara M, Ito K, Tanoue O, et al. Effective drug delivery system for duchenne muscular dystrophy using hybrid liposomes including gentamicin along with reduced toxicity. Biol. Pharm. Bull. 2011;34:712-716.

[134] Kaji H, Nagai N, Nishizawa M, et al. Drug delivery devices for retinal diseases. Adv. Drug Deliv. Rev. Elsevier B.V.; 2018. p. 148-157.

[135] Cao Y, Samy KE, Bernards DA, et al. Recent advances in intraocular sustained-release drug delivery devices. Drug Discov. Today. 2019;24:1694-1700.

[136] Duong TT, Lim J, Vasireddy V, et al. Comparative AAV-EGFP transgene expression using vector serotypes 1-9, 7M8, and $8 \mathrm{~b}$ in human pluripotent stem cells, RPEs, and human and rat cortical neurons. Stem Cells Int. 2019;2019.

[137] Matsuzono K, Imamura K, Murakami N, et al. Antisense Oligonucleotides Reduce RNA Foci in Spinocerebellar Ataxia 36 Patient iPSCs. Mol. Ther. - Nucleic Acids. 2017;8:211-219.

[138] Zhang Y, Sastre D, Wang F. CRISPR/Cas9 Genome Editing: A Promising Tool for Therapeutic Applications of Induced Pluripotent Stem Cells. Curr. Stem Cell Res. Ther. 2018;13:243-251.

[139] Wang D, Belakhov V, Kandasamy J, et al. The designer aminoglycoside NB84 significantly reduces glycosaminoglycan accumulation associated with MPS I-H in the Idua-W392X mouse. Mol. Genet. Metab. 2012;105:116-125.

[140] Cabrera FJ, Wang DC, Reddy K, et al. Challenges and opportunities for drug delivery to the posterior of the eye. Drug Discov. Today. 2019;24:1679-1684. 
